\title{
ChemComm
}

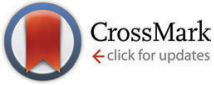

Cite this: Chem. Commun., 2015, 51,847

Received 28th October 2014, Accepted 21st November 2014

DOI: $10.1039 / c 4 c c 08513 b$

www.rsc.org/chemcomm

\section{A photoswitchable supramolecular complex with release-and-report capabilities $\dagger$}

\author{
Jesper R. Nilsson, ${ }^{a}$ Melanie C. O'Sullivan, ${ }^{b}$ S. Li, ${ }^{a}$ Harry L. Anderson ${ }^{b}$ and \\ Joakim Andréasson*a
}

\begin{abstract}
A self-assembled supramolecular platform has been designed for reversibly controlling the concentration of a compound in solution, via a photochemical reaction. The system utilizes metal-ligand interactions between a Zn-porphyrin dimer and a pyridine-appended dithienylethene (DTE) photoswitch. In addition to reversible compound release, the spectral properties of the release scaffold provide a fluorescence-based reporting function.
\end{abstract}

Controlling when and where a substance is released and/or captured has great potential in a wide range of applications, e.g. the release of therapeutic compounds, sensing agents, or extraction of hazardous chemicals and pollutants. The unique properties of light sets it aside as a triggering stimulus for applications requiring spatiotemporally well-resolved, waste-free operation. ${ }^{1}$ A number of approaches have been investigated in pursuit of light-operated release systems and these efforts have consequently stimulated a rapidly progressing and inventive research field. A majority of the so far reported light-controlled release systems, however, are designed to exhibit irreversible release. This is typically achieved by light-induced cleavage of covalent bonds either directly using photolabile groups/linkers ${ }^{2}$ or second-hand by first generating for instance heat. ${ }^{3}$ Another well explored approach is the preparation of light-responsive drug loaded materials such as porous materials ${ }^{4}$ and/or micro/nanoparticles. ${ }^{5}$

An additional level of control is attained if the photo-release can be made reversible, as it allows for dynamic bidirectional control of the concentration profile in combination with precise timing of the dosage. Realization of such systems typically demands a photoswitchable component capable of interacting non-covalently with a host compound to form a supramolecular complex. There are

\footnotetext{
${ }^{a}$ Department of Chemical and Biological Engineering, Chalmers University of Technology, SE-41296 Göteborg, Sweden. E-mail: a-son@chalmers.se; Fax: +46-31-772-3858; Tel: +46-31-772-2838

${ }^{b}$ Department of Chemistry, Oxford University, Oxford OX1 3TA, UK.

E-mail: harry.anderson@chem.ox.ac.uk; Fax: +44-(0)1865-2-85002; Tel: +44-(0)1865-2-75704

$\dagger$ Electronic supplementary information (ESI) available: Synthetic procedure for G-Pe, spectroscopic details. See DOI: 10.1039/c4cc08513b
}

several examples where this concept has been successfully implemented to manipulate for instance the release of small ions, ${ }^{6}$ as well as small molecules ${ }^{7}$ in a reversible manner. A particularly elegant example of photoreversible compound release was reported in the recent work by Clever and co-workers in which a photoswitchable coordination cage composed of Pd-coordinating dithienylethenes (DTEs) was shown to reversibly encapsulate inorganic guest molecules in response to light. $^{7 b}$

In this work, we report a conceptually different coordinationbased approach for compound (capture and) release. Here, the differences in binding mode and binding strength between the two isomeric forms of a pyridine-appended DTE photoswitch (1, see Fig. 1) and a porphyrin dimer $\left(\mathbf{P}_{2}\right)$ are combined into a self-assembled platform with release-and-report capabilities for lone pair-carrying guest molecules.

Since its discovery, the DTE-backbone has found regular use in photoswitching applications due to its renowned high degree of photoconversion, thermal stability, and resistance to photofatigue. ${ }^{8}$ The photoinduced ring-closing $(\mathbf{1 o} \rightarrow \mathbf{1 c})$ is achieved with $302 \mathrm{~nm}$ UV-light $\left(\Phi_{\mathrm{O} \rightarrow \mathrm{c}}=0.57\right)^{9}$ and converts the sample to virtually $100 \% \mathrm{1c}$. Subsequent visible light exposure $(\lambda>550 \mathrm{~nm})$ completely opens the sample to $1 \mathrm{o}\left(\Phi_{\mathrm{c} \rightarrow \mathrm{o}}=0.02\right)$. This switching cycle can be repeated several times without notable photodegradation ${ }^{10}$ (see Fig. S3 for absorption spectra of $\mathbf{1}$, and ESI $\dagger$ for details on isomerization quantum yield).

Porphyrin macrocycles have been included in numerous molecular and supramolecular constructs as building blocks with fluorescent, sensitizing, and/or energy/electron transfer capabilities. ${ }^{11}$ The two porphyrin units constituting the herein used $\mathbf{P}_{2}$ have a near-barrierless rotation around the central diethyne axis, thus allowing an even distribution of rotamers in the dimer. ${ }^{12}$ The aliphatic side chains ( $\mathrm{Ar}$ and $\mathrm{R}$ in Fig. 1) effectively prevent dimer stacking, as no aggregation was detected up to $\mathrm{mM}$ concentrations. As for coordination to $\mathbf{P}_{2}$, the two DTE-isomers 10 and $1 \mathrm{c}$ are notably different. The ringclosed isomer (1c) coordinates axially to one $\mathrm{Zn}$-center in $\mathbf{P}_{\mathbf{2}}$ by donating a pyridine electron lone pair. This complexation occurs in a consecutive $1: 1 \rightarrow 1: 2$ manner, with binding 
a)<smiles>Fc1ccc2sc3sc(-c4ccncc4)cc3c2c1</smiles>

10 $\stackrel{\text { UV light }}{\stackrel{\rightleftarrows}{\rightleftarrows}}$
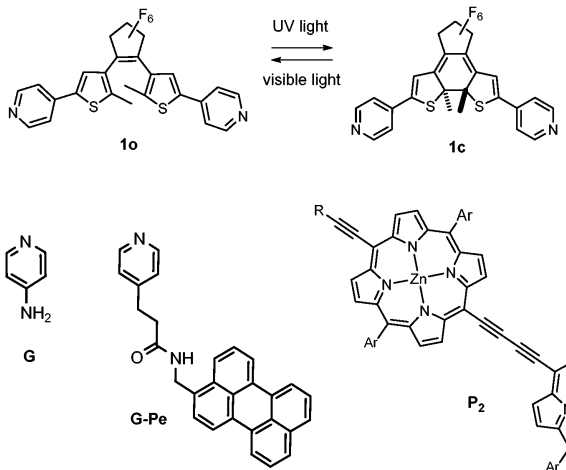

b)

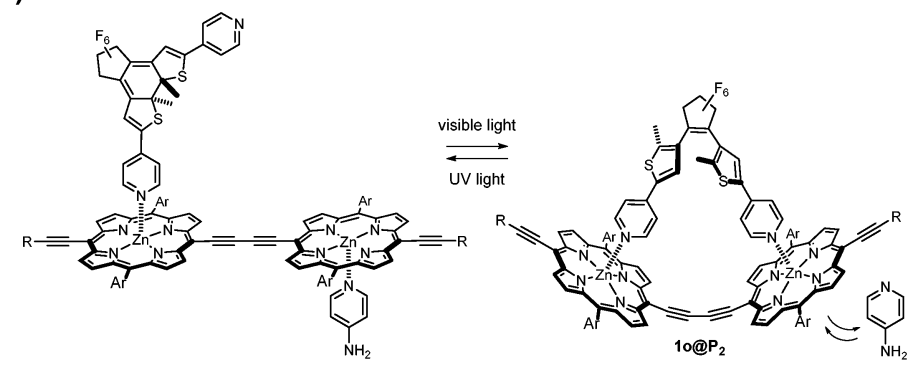

Fig. 1 (a) Isomerization scheme of 1 and molecular structures of 4-aminopyridine (G), the perylene functionalized analog (G-Pe), and the porphyrin dimer $\left(\mathbf{P}_{2}\right)$. (b) Photo-release principle. $\mathrm{Ar}=\mathrm{Si}\left(\mathrm{C}_{6} \mathrm{H}_{13}\right)_{3}, \mathrm{R}=3,5$-di(tert-butyl)phenyl.

constants of $K_{\mathrm{a} 1}=6.1 \times 10^{5} \mathrm{M}^{-1}$ and $K_{\mathrm{a} 2}=6.0 \times 10^{4} \mathrm{M}^{-1}$, respectively as determined by UV/Vis titrations (see $\mathrm{ESI} \dagger$ for titration details). For non-cooperative binding to a two-site host; $K_{\mathrm{a} 1}=4 \times K_{\mathrm{a} 2}$, indicating that binding of the second $1 \mathbf{c}$ to $\mathbf{P}_{2}$ exhibits slightly negative cooperativity, possibly due to minor steric interactions. In sharp contrast, the structural flexibility of the ring-opened isomer (10) allows it to stretch and instead form a 1:1 staple-like complex $\left(\mathbf{1 0} @ \mathrm{P}_{2}\right.$, Fig. 1). As a result of the double axial Zn-coordination, the latter binding is significantly stronger $\left(K_{\mathrm{a}}=5.5 \times 10^{6} \mathrm{M}^{-1}\right)$, in effect causing initially bound compounds to be released into solution as a result of competitive binding. The $10 @ \mathbf{P}_{2} 1: 1$ binding stoichiometry is strongly supported by the existence of no less than 8 isosbestic points throughout the 10 to $\mathbf{P}_{2}$ titration (Fig. S4, ESI $\dagger$ ). There is also a clear resemblance to the spectral changes seen upon $\mathbf{P}_{2}$ planarization using static (non-photochromic) ligands. ${ }^{12}$ Furthermore, the $1 \mathbf{1 0} \mathbf{P}_{\mathbf{2}}$ binding mode has been assessed by computational means. ${ }^{10}$ Binding of $\mathbf{1}$ to $\mathbf{P}_{\mathbf{2}}$ has no significant effect on the rate of the photoinduced ring-opening reaction, while the corresponding closing rate is reduced by a factor of 6 . This is likely due to coordination-induced restrictions in the conformational flexibility required for the isomerization process to occur. The usefulness of combining photoswitchable units and metalloporphyrins/porphyrinoids in supramolecular strategies is evidenced by the wide variety of processes brought under reversible photonic control using these building blocks. These include emission intensity, ${ }^{13}$ energy transfer, ${ }^{14}$ electron transfer, ${ }^{15}$ magnetic properties, ${ }^{16}$ and singlet oxygen generation. ${ }^{17}$

Here, the drug chosen to illustrate the release event is the well known small molecule neurotransmitter 4-aminopyridine (G, see Fig. 1).$^{18}$ In principle, any monodentate Lewis base can be used, the main prerequisite being adequate coordination capabilities (i.e. suitable binding strength) to $\mathbf{Z n}$ in $\mathbf{P}_{\mathbf{2}}$. However, any change in the UV/Vis absorption of $\mathbf{G}$ upon coordination to $\mathbf{P}_{\mathbf{2}}$ is obscured by the corresponding changes of the latter. Hence, a more straightforward means of monitoring binding/release of $\mathbf{G}$ is needed. Therefore, G-Pe was synthesized as a fluorescent model compound. G and G-Pe have identical binding modes to $\mathbf{P}_{2}$. The binding constants are: $K_{\mathrm{a} 1}=$ $3.2 \times 10^{5} \mathrm{M}^{-1}, K_{\mathrm{a} 2}=1.2 \times 10^{5} \mathrm{M}^{-1}$ and $K_{\mathrm{a} 1}=1.3 \times 10^{5} \mathrm{M}^{-1}, K_{\mathrm{a} 2}=$ $3.6 \times 10^{4} \mathrm{M}^{-1}$ for $\mathbf{G}$ and G-Pe respectively. The choice of the perylene fluorophore is motivated by the excellent spectral overlap between the emission spectra of G-Pe, and the $\mathbf{P}_{2}$ Soret band. Hence, binding to $\mathbf{P}_{\mathbf{2}}$ efficiently quenches the G-Pe emission by excitation energy transfer (EET, $R_{0}=66 \AA$, see Fig. S12 for details, ESI $\dagger$ ), possibly in combination with electron transfer (ET). Accordingly, the observed G-Pe emission originates exclusively from compound free in solution. It should be noted that G-Pe has nothing to do with the function of the release scaffold per se; it is used merely as a tool for monitoring the release.

The release of G-Pe is demonstrated in Fig. 2, where an initial cocktail of $\mathbf{P}_{2}, \mathbf{1 0}$, and $\mathbf{G - P e}$ gives rise to high emission, as the strongly coordinating 10 occupies both $\mathrm{Zn}$ binding-sites in $\mathbf{P}_{\mathbf{2}}$. Subjecting the solution to UV-light causes a $1 \mathbf{0} \rightarrow \mathbf{1 c}$ isomerization, whereafter each DTE-unit cannot coordinate more than one Zn-center. In response, G-Pe binds to the liberated coordination site,

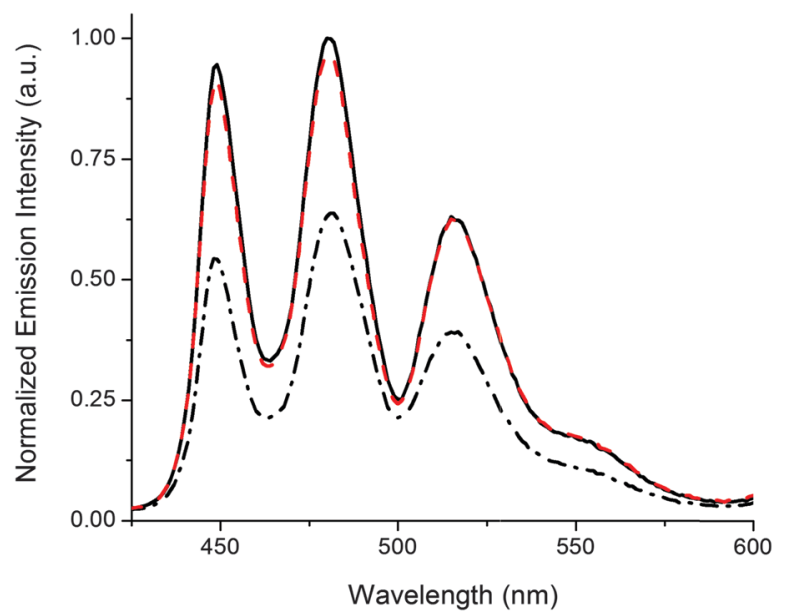

Fig. 2 Reversible release of G-Pe in response to light monitored by the emission intensity of the uncomplexed G-Pe population. Applied concentrations; $\left[\mathbf{P}_{\mathbf{2}}\right]=280 \mu \mathrm{M},[\mathbf{1}]=300 \mu \mathrm{M},[\mathbf{G}-\mathbf{P e}]=1 \mu \mathrm{M}$. Initially, $\mathbf{1}$ is in the open form 1o (solid black line). 2 min 302 nm UV-exposure triggers isomerization to $1 \mathrm{c}$ (dash-dotted black line). Subsequent visible light isomerizes the sample back to 10 ( $\lambda>550 \mathrm{~nm}, 3 \mathrm{~min}$, dashed red line). Please note that the experimental conditions/setup allows emission intensities unaffected by isomerization-induced inner filter effects. $\ddagger$ 


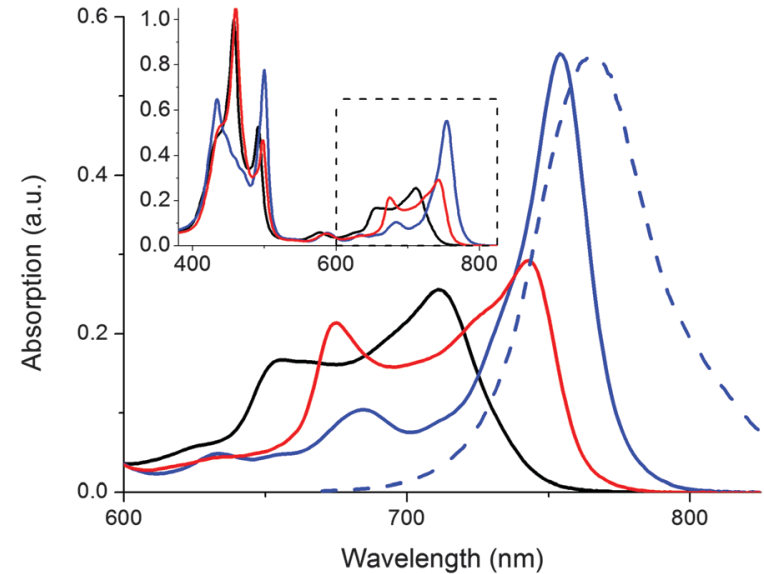

Fig. 3 Absorption spectra of supramolecular $\mathbf{P}_{\mathbf{2}}$-complexes in toluene: $\mathbf{P}_{\mathbf{2}}$

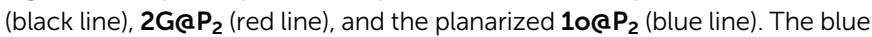
dashed line shows the $10 \mathrm{aP}_{2}$ emission spectra $\left(\lambda_{\text {exc }}=510 \mathrm{~nm}\right)$. Inset: Absorption from $380-825 \mathrm{~nm}$.

and the emission is quenched. Subsequent visible light restores the high emission by reforming 10 and displacement of G-Pe from $\mathbf{P}_{2}$.

In addition to triggering the compound release, formation of the doubly coordinated 10@P $\mathbf{P}_{2}$ complex forces the two porphyrin macrocycles to adopt a coplanar conformation. With this restriction in $\mathbf{P}_{2}$ rotameric distribution comes characteristic changes in the absorption spectrum (Fig. 3).

In Fig. 3, the absorption spectral signatures of $\mathbf{P}_{\mathbf{2}}$ and the two types of $\mathbf{P}_{2}$ complexes are shown. Monodentate species typically induce a red-shift in the $\mathbf{P}_{2}$ Q-band (711 nm $\left.\rightarrow \sim 745 \mathrm{~nm}\right)$. The planar complex (10@P $\mathbf{P}_{2}$ ) exhibits a further red shifted, and significantly hyperchromic absorption band centered at $754 \mathrm{~nm}$. The spectral features inherent to the rotational distribution of diethynelinked porphyrin dimers and oligomers have been used to control the rate of electron transfer ${ }^{19}$ as well as singlet oxygen generation, ${ }^{20}$ by allowing selective excitation of planar or randomly oriented rotamers. In our laboratory, we have devised a molecular memory capable of non-destructive readout based on photochromic planarization of a porphyrin dimer. ${ }^{10}$ Here, as the compound release proceeds concurrently with a marked increase in absorption of $\mathbf{P}_{\mathbf{2}}$ around $750 \mathrm{~nm}$, it is possible to read the state of the release scaffold to confirm the release event by probing the emission at $800 \mathrm{~nm}$, following excitation at $790 \mathrm{~nm}$. Hence, the inherent fluorescent properties of the scaffold are in line with the so-called release-and-report function.

The typical "release" (e.g. caged compounds ${ }^{2 b}$ ) requires UV-light. For most light-controlled applications, this is not optimal, due to limited penetration depth and potential damage to surrounding tissue, materials, and/or the released compound itself. Here, a notable advantage is that both the release- and the report functions are triggered by low energy photons ( $\lambda$ up to $c a$. $700 \mathrm{~nm}$ and almost $800 \mathrm{~nm}$, respectively). To illustrate the performance and stability of the release scaffold, we prepared a sample containing $\mathbf{P}_{\mathbf{2}}, \mathbf{1 0}$, and $\mathbf{G}$, and subjected the solution to alternating irradiation of $302 \mathrm{~nm}$ and visible light $(\lambda>550 \mathrm{~nm})$, probing the report output after each irradiation step (Fig. 4).

It is clear that $1 \mathrm{c} \rightleftharpoons \mathbf{1 0}$ isomerization causes dramatic differences in emission intensity from $\mathbf{P}_{2}$, and that switching

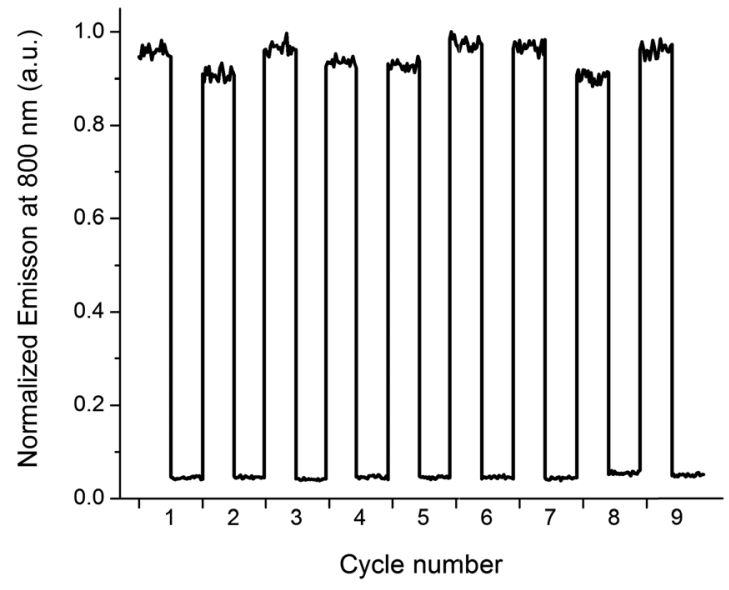

Fig. 4 Photoswitching performance of the release-and-report system, as monitored via the report function, i.e. emission intensity at $800 \mathrm{~nm}\left(\lambda_{\mathrm{exc}}=\right.$ $790 \mathrm{~nm}$ ). Applied concentrations; $\left[\mathbf{P}_{\mathbf{2}}\right]=280 \mu \mathrm{M},[\mathbf{1}]=300 \mu \mathrm{M},[\mathbf{G}]=1 \mu \mathrm{M}$. Each cycle starts with 1 in the open form 10 (high intensity). 2 min 302 nm UV-exposure triggers isomerization to $1 \mathrm{c}$ (low intensity). Subsequent visible light ( $\lambda>550 \mathrm{~nm}, 3 \mathrm{~min}$ ) isomerizes the sample back to 10

between the two types of complexes is fully reversible, and proceeds with no apparent photodegradation.

The authors appreciate the limited biological compatibility of the solvents used throughout this proof-of-principle study. Thus, for use in biological environments, solubilization of the release scaffold needs to be addressed. In this context it deserves mentioning that metal-ligand coordination approaches to photo-release (albeit irreversible) in living organisms have been reported, ${ }^{21}$ along with examples of porphyrin dimers ${ }^{22}$ and DTEs ${ }^{23}$ adapted for, and used in, biological applications.

A self-assembled system for reversible photo-control of compound release has been demonstrated. The unique spectral properties inherent to this system conveniently allows for fluorescence-based assessment of the state of the release scaffold, i.e. whether the compound is bound or not. This reporter ability has to our knowledge not been demonstrated in a reversible release system to date; thus this work represents a conceptually valuable addition to existing photo-operated release systems. The affinity of Zn-porphyrins for amine-based ligands implies that this reversible release system could be applied to a wide range of ligands, eliminating the need for guest-specific synthetic efforts.

This work was financed by the Swedish Research Council (Grant 622-2010-280) and the European Research Council (ERC FP7/2007-2013 Grant No. 203952). Professor Neil Branda is acknowledged for valuable discussions.

\section{Notes and references}

$\ddagger$ Under the selected conditions, $[\mathbf{1}]$ and $\left[\mathbf{P}_{2}\right]$ give rise to a significant optical density in the solution. It should therefore be noted that the emission measurements were performed in a $1 \mathrm{~mm}$ cuvette with front-face detection and excitation at an isosbestic point, $\lambda_{\mathrm{exc}}=$ $410 \mathrm{~nm}$.

1 R. Göstl, A. Senf and S. Hecht, Chem. Soc. Rev., 2014, 43, 1982.

2 (a) K. L. Ciesienski and K. J. Franz, Angew. Chem., Int. Ed., 2011, 50, 814; (b) C. Brieke, F. Rohrbach, A. Gottschalk, G. Mayer and A. Heckel, Angew. Chem., Int. Ed., 2012, 51, 8446; (c) P. Klán, 
T. Šolomek, C. G. Bochet, A. Blanc, R. Givens, M. Rubina, V. Popik, A. Kostikov and J. Wirz, Chem. Rev., 2012, 113, 119; (d) C. G. Bochet, J. Chem. Soc., Perkin Trans. 1, 2002, 125.

3 (a) A. B. S. Bakhtiari, D. Hsiao, G. Jin, B. D. Gates and N. R. Branda, Angew. Chem., Int. Ed., 2009, 48, 4166; (b) W. F. Zandberg, A. B. S. Bakhtiari, Z. Erno, D. Hsiao, B. D. Gates, T. Claydon and N. R. Branda, J. Nanomed. Nanotechnol., 2012, 8, 908.

4 (a) N. K. Mal, M. Fujiwara, Y. Tanaka, T. Taguchi and M. Matsukata, Chem. Mater., 2003, 15, 3385; (b) E. Aznar, M. D. Marcos, R. MartínezMáñez, F. Sancenón, J. Soto, P. Amorós and C. Guillem, J. Am. Chem. Soc., 2009, 131, 6833; (c) J. Lu, E. Choi, F. Tamanoi and J. I. Zink, Small, 2008, 4, 421; (d) R. Tong, H. D. Hemmati, R. Langer and D. S. Kohane, J. Am. Chem. Soc., 2012, 134, 8848.

5 (a) H. Yan, C. Teh, S. Sreejith, L. Zhu, A. Kwok, W. Fang, X. Ma, K. T. Nguyen, V. Korzh and Y. Zhao, Angew. Chem., Int. Ed., 2012, 51, 8373; (b) M. S. Yavuz, Y. Cheng, J. Chen, C. M. Cobley, Q. Zhang, M. Rycenga, J. Xie, C. Kim, K. H. Song, A. G. Schwartz, L. V. Wang and Y. Xia, Nat. Mater., 2009, 8, 935; (c) W. Xiao, W.-H. Chen, J. Zhang, C. Li, R.-X. Zhuo and X.-Z. Zhang, J. Phys. Chem. B, 2011, 115, 13796.

6 (a) M. Blank, L. Soo, H. Wassermann and B. Erlanger, Science, 1981, 214, 70; (b) S. Lee and A. H. Flood, J. Phys. Org. Chem., 2013, 26, 79.

7 (a) H. Dube and J. Rebek, Angew. Chem., Int. Ed., 2012, 51, 3207; (b) M. Han, R. Michel, B. He, Y.-S. Chen, D. Stalke, M. John and G. H. Clever, Angew. Chem., Int. Ed., 2013, 52, 1319; (c) Y. Wang, M. Zhang, C. Moers, S. Chen, H. Xu, Z. Wang, X. Zhang and Z. Li, Polymer, 2009, 50, 4821; (d) G. H. Clever, S. Tashiro and M. Shionoya, J. Am. Chem. Soc., 2010, 132, 9973.

8 M. Irie, Chem. Rev., 2000, 100, 1685.
9 Quantum yield for the ring-closing reaction was determined in methanol in; K. Matsuda, Y. Shinkai, T. Yamaguchi, K. Nomiyama, M. Isayama and M. Irie, Chem. Lett., 2003, 32, 1178.

10 J. Kärnbratt, M. Hammarson, S. Li, H. L. Anderson, B. Albinsson and J. Andréasson, Angew. Chem., Int. Ed., 2010, 49, 1854.

11 (a) I. Beletskaya, V. S. Tyurin, A. Y. Tsivadze, R. Guilard and C. Stern, Chem. Rev., 2009, 109, 1659; (b) C. A. Hunter and R. K. Hyde, Angew. Chem., Int. Ed., 1996, 35, 1936.

12 M. U. Winters, J. Kärnbratt, M. Eng, C. J. Wilson, H. L. Anderson and B. Albinsson, J. Phys. Chem. C, 2007, 111, 7192.

13 (a) T. B. Norsten and N. R. Branda, Adv. Mater., 2001, 13, 347; (b) J. Otsuki and K. Narutaki, Bull. Chem. Soc. Jpn., 2004, 77, 1537; (c) D. R. Reddy and B. G. Maiya, Chem. Commun., 2001, 117.

14 J. Otsuki, A. Yasuda and T. Takido, Chem. Commun., 2003, 608.

15 A. J. Myles and N. R. Branda, J. Am. Chem. Soc., 2000, 123, 177.

16 S. Thies, H. Sell, C. Bornholdt, C. Schütt, F. Köhler, F. Tuczek and R. Herges, Chem. - Eur. J., 2012, 18, 16358.

17 L. Hou, X. Zhang, T. C. Pijper, W. R. Browne and B. L. Feringa, J. Am. Chem. Soc., 2014, 136, 910.

18 M. Müller, P. W. Dierkes and W.-R. Schlue, Brain Res., 1999, 826, 63.

19 M. U. Winters, J. Kärnbratt, H. E. Blades, C. J. Wilson, M. J. Frampton, H. L. Anderson and B. Albinsson, Chem. - Eur. J., 2007, 13, 7385.

20 M. K. Kuimova, M. Balaz, H. L. Anderson and P. R. Ogilby, J. Am. Chem. Soc., 2009, 131, 7948.

21 L. Zayat, C. Calero, P. Alborés, L. Baraldo and R. Etchenique, J. Am. Chem. Soc., 2003, 125, 882.

22 M. K. Kuimova, S. W. Botchway, A. W. Parker, M. Balaz, H. A. Collins, H. L. Anderson, K. Suhling and P. R. Ogilby, Nat. Chem., 2009, 1, 69.

23 T. C. S. Pace, V. Müller, S. Li, P. Lincoln and J. Andréasson, Angew. Chem., Int. Ed., 2013, 52, 4393. 